\title{
Efeitos da leitura, exercício e exercício sob leitura na pressão intra-ocular de portadores de glaucoma primário de ângulo aber to ou hipertensão ocular controlados clinicamente com medicação tópica
}

\author{
Effects of reading, exercise and exercise combined with reading on intraocular \\ pressure for patients sustaining primary glaucoma (open angle) or ocular \\ hypertension, both clinically controlled with topic medication
}

\author{
Alice Maria Corrêa Medina ${ }^{1}$ \\ Núbia Vanessa dos Anjos Lima² \\ Regiina Cândida Ribeiro dos Santos ${ }^{3}$ \\ Marco César Araújo Pereira ${ }^{4}$ \\ Procópio Miguel dos Santos ${ }^{5}$
}

Trabalho realizado no Hospital de Base do Distrito Federal (HBDF) - UnB e na Clínica OFTALMED Brasília (DF) - Brasil.

${ }^{1}$ Pós-Graduanda (Nível Doutorado) da Universidade de Brasília - UnB - Brasília (DF) - Brasil.

${ }^{2}$ Especialista em Glaucoma pelo Hospital São Geraldo da Universidade Federal de Minas Gerais - UFMG Belo Horizonte (MG) - Brasil.

${ }^{3}$ Professora Orientadora do Curso da Pós-Graduação da UnB - Brasília (DF) - Brasil.

${ }^{4}$ Mestre em Estatística pela UnB - Brasília (DF) - Brasil.

${ }^{5}$ Professor Orientador do Curso da Pós-Graduação da UnB - Brasília (DF) - Brasil.

Endereço para correspondência: Alice Maria Corrêa Medina. SQN 412, B1. K, Apto. 101 - Brasília (DF) CEP 70867-110

Recebido para publicação em 10.06.2005

Última versão recebida em 14.04.2006

Aprovação em 13.05.2006

Dados parciais da tese a ser apresentada ao curso de Pós-Graduação em Ciências da Saúde, Universidade de Brasília - UnB, área de concentração oftalmologia, para obtenção do título de Doutor.

Nota Editorial: Depois de concluída a análise do artigo sob sigilo editorial e com a anuência dos Drs. Ruth Rosenhek Schor e Francisco Eduardo Lopes de Lima sobre a divulgação de seus nomes como revisores, agradecemos sua participação neste processo.

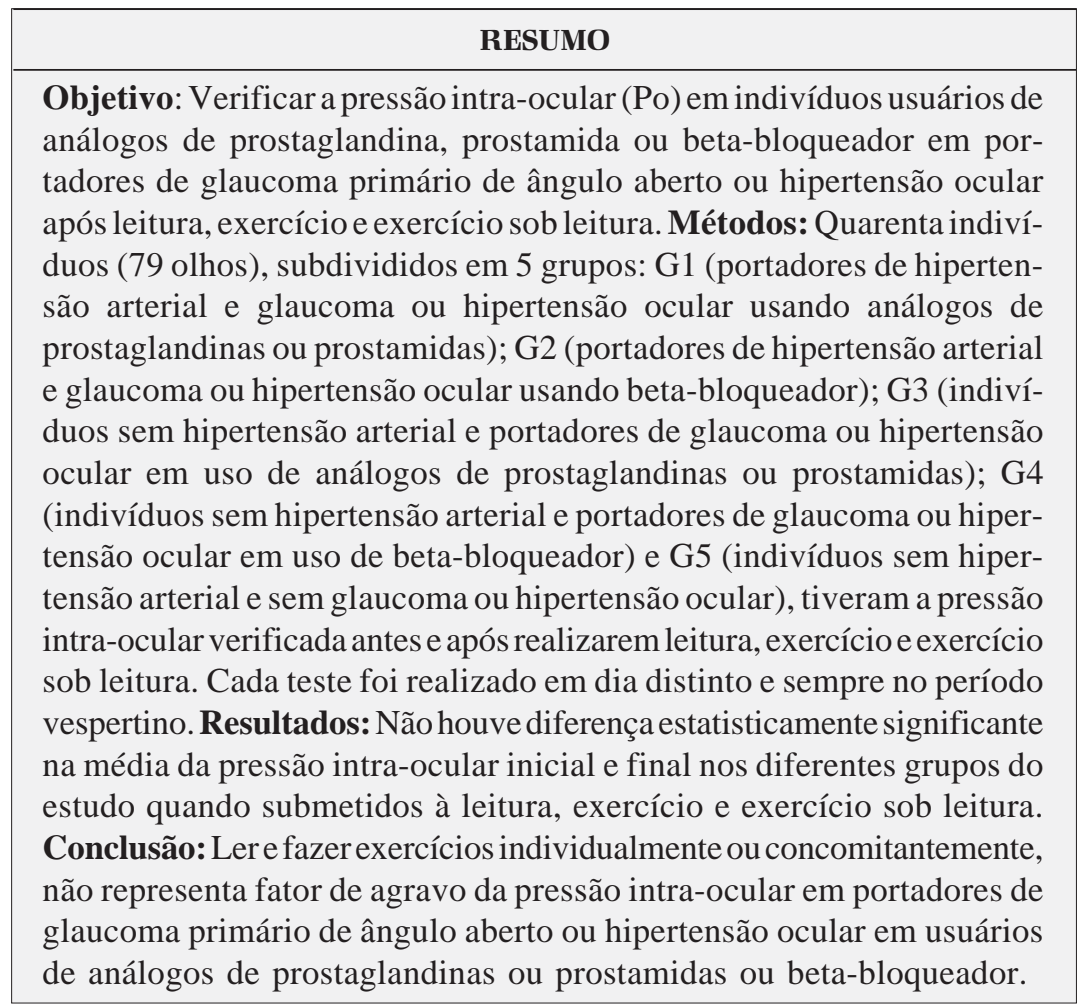

Descritores: Glaucoma de ângulo aberto; Pressão intra-ocular/quimioterapia; Leitura; Exercício; Hipertensão ocular; Prostaglandinas/uso terapêutico; Tonometria ocular

\section{INTRODUÇÃO}

Durante a acomodação para leitura, a contração do músculo ciliar afrouxa a tensão do ligamento o que permite ao cristalino ficar mais globular, permitindo assim o foco, ocorrendo redução da pressão intra-ocular $(\mathrm{Po})^{(1-2)}$.

De um modo geral, não há restrições à prática de exercícios físicos para pacientes com glaucoma primário de ângulo aberto em bicicleta ergométrica, onde foi comprovado que exercícios físicos reduzem a $\mathrm{Po}^{(3)}$. Indivíduos sedentários e saudáveis obtiveram uma redução da Po similares em ambos 
116 Efeitos da leitura, exercício e exercício sob leitura na pressão intra-ocular de portadores de glaucoma primário de ângulo aberto ou hipertensão ocular controlados clinicamente com medicação tópica

os olhos, após exercícios físicos amenos, moderados e intensos, onde se conclui que há uma diminuição da Po após todas as formas de exercícios ${ }^{(4)}$. Em algumas pesquisas realizadas foram constatadas diferenças significativas da Po ao realizar exercícios com intensidades mais elevadas ${ }^{(5-6)}$.

Em um estudo, no qual o objetivo foi avaliar os efeitos dos exercícios de força sobre a Po de homens e mulheres não glaucomatosos e ativos fisicamente, os resultados apresentados demonstraram que ocorre uma redução da Po em ambos os gêneros ${ }^{(7)}$.

Segundo um estudo realizado com indivíduos com hipertensão arterial sistêmica e diabetes mellitus, observou-se que o valor médio da Po é progressivamente mais elevado quando se considera o tempo de instalação da doença hipertensiva sistêmica, cujo quadro e evolução são também fatores que contribuem para este aumento, principalmente quando associada a diabetes ${ }^{(8)}$.

A medicação tópica como os análogos das prostaglandinas, prostaminas e beta-bloqueadores são drogas que vêm desempenhando um importante papel no controle do paciente glaucomatoso $^{(9)}$.

O objetivo do presente trabalho foi verificar os efeitos da leitura, do exercício e do exercício sob leitura em pacientes com glaucoma primário de ângulo aberto ou hipertensos oculares usuários de substâncias com princípio ativo de prostaglandina ou beta-bloqueador.

\section{MÉTODOS}

A pesquisa foi realizada nos meses de junho, julho, outubro e novembro de 2004 na Clínica Oftalmed em local reservado para os testes, onde os dados foram coletados pelos mesmos profissionais, utilizando-se os mesmos instrumentos para todos os pacientes e indivíduos sadios.

Foram selecionadas as seguintes variáveis: sexo, idade, normotensão sistêmica, hipertensão sistêmica, uso de colírios de análogos de prostaglandinas, prostamidas e beta-bloqueador.

O referido estudo foi aprovado pelo Comitê de Ética e Pesquisa da Universidade de Brasília (UnB), em 09/03/2004 sob processo $\mathrm{n}^{\circ}$ 007/2004.

Os participantes foram informados sobre o caráter experimental e concordaram em assinar um termo de consentimento.

Para o presente estudo, foram selecionados pacientes de 40 a 72 anos de ambos os sexos, portadores de glaucoma primário de ângulo aberto ou hipertensão ocular utilizando medicação antiglaucomatosa na forma de colírios há mais de um mês. Todos os pacientes tinham diagnóstico de glaucoma primário de ângulo aberto ou hipertensão ocular e estavam sendo tratados por médico especialista em glaucoma, onde aqueles pertencentes aos grupos com hipertensão sistêmica tinham a pressão arterial controlada com medicação.

Os indivíduos foram distribuídos em 5 grupos de 8 indivíduos cada, seguindo o diagnóstico clínico-oftalmológico e o princípio ativo do colírio utilizando conforme o esquema abaixo:
Grupo 1: portadores de hipertensão arterial e glaucoma ou hipertensão ocular usando análogos de prostaglandinas ou prostamidas;

Grupo 2: portadores de hipertensão arterial e glaucoma ou hipertensão ocular usando beta-bloqueador;

Grupo 3: indivíduos sem hipertensão arterial e portadores de glaucoma ou hipertensão ocular em uso de análogos de prostaglandinas ou prostamidas;

Grupo 4: indivíduos sem hipertensão arterial e portadores de glaucoma ou hipertensão ocular em uso de beta-bloqueador;

Grupo 5: indivíduos sem hipertensão arterial e sem glaucoma ou hipertensão ocular sem uso de colírio hipotensor.

Todos os pacientes do grupo 1 ao 4 faziam uso de apenas um colírio antiglaucomatoso que poderia ser:

Bimatoprost $0,03 \%$ (Lumigan $\left.{ }^{\circledR}\right)$, Travoprost $\left(\right.$ Travatan $\left.^{\circledR}\right)$, Latanoprost $\left(\right.$ Xalatan $\left.^{\circledR}\right)$, Maleato de timolol 0,5\% (Timoptol $0,5 \%{ }^{\circledR}$ ), Maleato de timolol $0,25 \%$ (Timoptol $0,25 \%{ }^{\circledR}$ ), Maleato de Timolol 0,1\% $\left(\mathrm{Nyolol}^{\circledR}\right)$, Betaxolol 0,5\% (Presmin) e tinham a Po $\leq 18 \mathrm{mmHg}$ usando a medicação.

Todos os indivíduos envolvidos no estudo realizaram três testes: leitura, exercício e exercício sob leitura durante $15 \mathrm{mi}-$ nutos em bicicleta ergométrica realizados em dias diferentes, sempre no período vespertino, pois durante o período matutino há uma maior variabilidade da Po. A distância para leitura foi de $33 \mathrm{~cm}$ entre os olhos do leitor e o painel da bicicleta, onde a revista foi fixada.

As coletas de dados das variáveis: Po, pressão arterial sistêmica (PA) e freqüência cardíaca (FC) foram realizadas em cada participante no início e final de cada teste. Após o término dos referidos testes, os participantes permaneceram sentados na bicicleta durante o tempo de um minuto, sendo encaminhados posteriormente para uma outra sala para aferição da Po.

Barros (2002) classifica como sedentário o indivíduo que não pratica exercícios físicos regulares com a freqüência mínima de 3 vezes por semana, e indica a fórmula para o cálculo da frequiência cardíaca máxima (FC) para sedentários: $\mathrm{FC}=208$ $0,7 \mathrm{x}$ idade. A zona-alvo foi definida com o intervalo de $60 \%$ a $70 \%$ da FC máxima ${ }^{(10)}$.

A determinação e o monitoramento da intensidade dos exercícios foram monitorados nas respectivas zonas de trabalho (zona-alvo).

Os indivíduos foram informados, que caso ocorresse algum desconforto, como mal-estar, tonturas, etc, os testes seriam interrompidos sem nenhum ônus para os mesmos. Após a acomodação do paciente na bicicleta e utilizando um freqüencímetro cardíaco para monitoramento da freqüência cardíaca (FC), a carga foi introduzida progressivamente até atingir o limite inferior de $60 \%$ da FC máxima, iniciando-se a contabilização do tempo de teste, 15 minutos. Cada paciente manteve-se na sua respectiva zona de trabalho durante este tempo, a qual compreendeu uma faixa de $60 \%$ a $70 \%$ da FC máxima calculada.

O protocolo utilizado para o exercício físico de $60 \%$ a $70 \%$ da FC máxima, foi baseado no perfil dos participantes do estudo, sedentários com ou sem hipertensão arterial sistêmica, não praticante de exercício físico de forma regular no mínimo três vezes por semana em academias ou clubes. 
Houve recomendações prévias aos pacientes para que não ingerissem bebidas alcoólicas 24 horas antes dos testes, assim como vestuário apropriado para a prática dos exercícios físicos.

Para este estudo, utilizou-se o sistema duplo-cego, onde a verificação da Po foi realizada sem o conhecimento do oftalmologista sobre o tipo de teste realizado naquele dia, sendo a verificação da mesma realizada em outro ambiente. A pressão intra-ocular foi aferida utilizando-se um tonômetro de aplanação Goldmann.

Foi realizado o tratamento estatístico do grupo usando-se o teste ANOVA, teste $t$ de Student, (pareado para avaliação intragrupos), em estudo experimental, duplo cego randomizado. $\mathrm{O}$ tratamento estatístico utilizado foi adequado ao número de participantes distribuídos em 5 grupos de 8 , com um total de 40 indivíduos.

Optou-se por um estudo de blocos inteiramente ao acaso. Caso as diferenças fossem significativas teria sido usado o teste Post-Hoc (Tukey) para saber em que teste houve essa diferença. O nível de significância estabelecido foi de $p<0,05$.

A aferição da PA, assim como a determinação da FC de trabalho, variáveis descritivas, foram utilizadas como controle nos testes. O estudo teve como variáveis independentes a leitura e o exercício e dependente a pressão intra-ocular.

\section{RESULTADOS}

A idade dos indivíduos estudados variou de 40 a 72 anos com uma mediana de 54 anos, (a mediana foi utilizada por ser uma medida de centralização mais robusta do que a média). $\mathrm{O}$ estudo contou com 26 participantes do sexo feminino e 14 do sexo masculino.

Foram realizadas seis análises de regressão com a Po inicial e final do olho direito (OD) e olho esquerdo (OE) em cada um dos tratamentos para os testes de leitura (T1), exercício (T2) e exercício sob leitura (T3). A finalidade foi confirmar a hipótese de que não há diferença na pressão intra-ocular devido à lateralidade do olho. Se a pressão independe da lateralidade, então os valores devem se distribuir como a bissetriz do primeiro quadrante, isto é, uma reta do tipo $\mathrm{y}=\mathrm{x}$. Todas as regressões confirmaram esta hipótese $(\mathrm{p}<1 \%)$.

Após a realização do teste ANOVA, uma via, no qual se correlacionou cada grupo isoladamente com o grupo controle, foram obtidos os valores da Po, utilizando o nível de significância de $5 \%$.
Pelos resultados obtidos, não houve diferença significante entre a Po do olho direito e do olho esquerdo, onde foi utilizada então a média da Po para cada indivíduo. Realizou-se uma análise de variância de blocos inteiramente ao acaso, com os testes (T1, T2 e T3), pressão arterial (hipertensão sistêmica e normotensos), tipo de colírio (beta-bloqueador ou análogos de prostaglandina, prostamida ou controle) e fase dos testes (inicial e final). Foram realizados tratamentos para efetuar a análise de variância, onde os resultados demonstraram que não foi encontrada diferença significante entre os tipos de tratamentos. Os valores de média e desvio-padrão iniciais e finais nos testes (T1, T2 e T3) dos grupos encontram-se na tabela 1.

Os resultados demonstraram que ao se comparar as médias iniciais de todos os grupos com as médias finais, observou-se uma diminuição da Po após o teste de leitura (T1), sem diferença significante entre os grupos. No teste de exercício (T2) observou-se também uma redução da Po final em relação a inicial em todos os grupos, sem haver diferenças entre os grupos de participantes.

No teste de exercício sob leitura (T3) a Po final manteve igual a inicial no grupo G2 (hipertensos sistêmicos utilizando beta-bloqueador) e diminuiu em relação a inicial para os demais grupos (Tabela 1).

A tabela 1 mostra os valores das médias e dos desviospadrão observado nas duas fases (inicial e final) para cada um dos testes efetuados (T1, T2 e T3), para cada um dos grupos estudados.

Os gráficos 1, 2 e 3 mostram as médias em cada grupo, bem como o respectivo intervalo com um desvio padrão.

\section{DISCUSSÃO}

Estudos com indivíduos normais, demonstraram que a pressão intra-ocular diminuiu durante exercícios ${ }^{(11)}$ e ao utilizar exercícios submáximos com cargas ${ }^{(12)}$. Em pacientes com glaucoma primário de ângulo aberto realizando caminhadas, a Po diminuiu ${ }^{(13)}$ em relação às médias iniciais.

As análises realizadas neste estudo demonstraram que não houve diferença significante da Po em relação à lateralidade do olho direito (OD) e olho esquerdo (OE) para os testes de leitura (T1), exercício (T2) e exercício sob leitura (T3) na Po inicial e final dos 5 grupos ( $\mathrm{p}<1 \%)$, sendo utilizada as médias das respectivas pressões iniciais e finais. Nos testes de leitura

\begin{tabular}{|c|c|c|c|c|c|c|c|c|c|c|c|c|}
\hline \multirow{4}{*}{ Grupo } & \multirow{2}{*}{\multicolumn{4}{|c|}{ T1 }} & \multirow{2}{*}{\multicolumn{4}{|c|}{ T2 }} & \multirow{2}{*}{\multicolumn{4}{|c|}{ T3 }} \\
\hline & & & & & & & & & & & & \\
\hline & \multicolumn{2}{|c|}{ Inicial } & \multicolumn{2}{|c|}{ Final } & \multicolumn{2}{|c|}{ Inicial } & \multicolumn{2}{|c|}{ Final } & \multicolumn{2}{|c|}{ Inicial } & \multicolumn{2}{|c|}{ Final } \\
\hline & Média & DP & Média & $\overline{D P}$ & Média & $\overline{D P}$ & Média & DP & Média & $\overline{D P}$ & Média & DP \\
\hline G1 & 14,25 & 2,27 & 13,50 & 2,58 & 14,25 & 1,91 & 12,75 & 1,73 & 13,31 & 2,02 & 12,88 & 2,25 \\
\hline G2 & 14,13 & 1,55 & 13,47 & 1,46 & 13,80 & 1,78 & 12,33 & 1,72 & 13,73 & 1,67 & 13,73 & 1,44 \\
\hline G3 & 13,69 & 2,36 & 12,56 & 1,41 & 13,13 & 2,25 & 13,00 & 2,71 & 13,69 & 2,27 & 13,31 & 1,66 \\
\hline G4 & 13,00 & 1,63 & 12,00 & 1,15 & 13,25 & 1,44 & 12,25 & 1,29 & 13,31 & 1,66 & 12,88 & 2,09 \\
\hline G5 & 12,88 & 2,22 & 12,31 & 2,41 & 12,56 & 2,39 & 12,13 & 2,33 & 12,38 & 2,06 & 12,00 & 2,19 \\
\hline
\end{tabular}


118 Efeitos da leitura, exercício e exercício sob leitura na pressão intra-ocular de portadores de glaucoma primário de ângulo aberto ou hipertensão ocular controlados clinicamente com medicação tópica

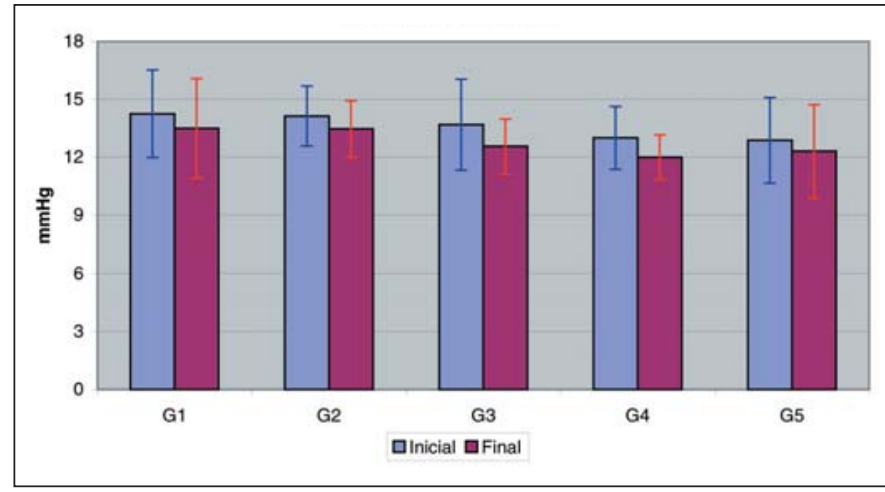

Gráfico 1 - Valores das médias da Po $(\mathrm{mmHg})$ iniciais e finais nos cinco grupos do teste $\mathrm{T} 1$ e respectivos intervalos de um desvio padrão

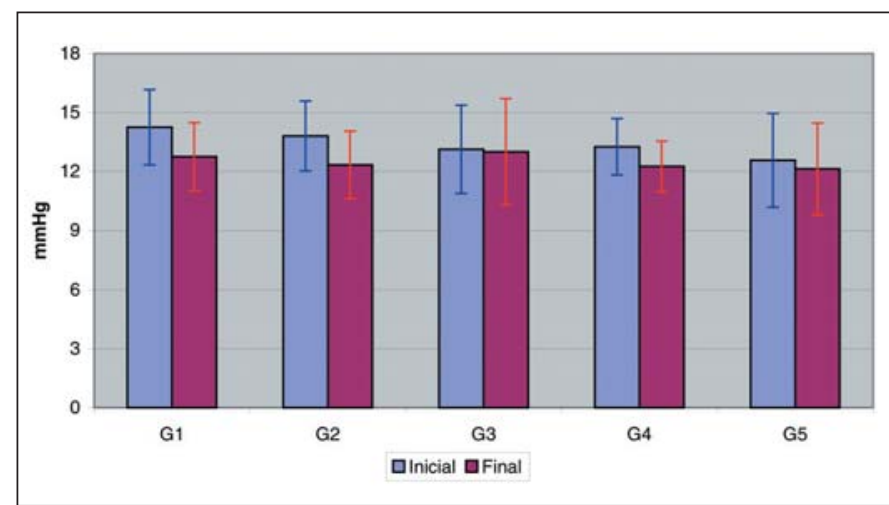

Gráfico 2 - Valores das médias da Po $(\mathrm{mmHg})$ iniciais e finais nos cinco grupos do teste $\mathrm{T} 2$ e respectivos intervalos de um desvio padrão

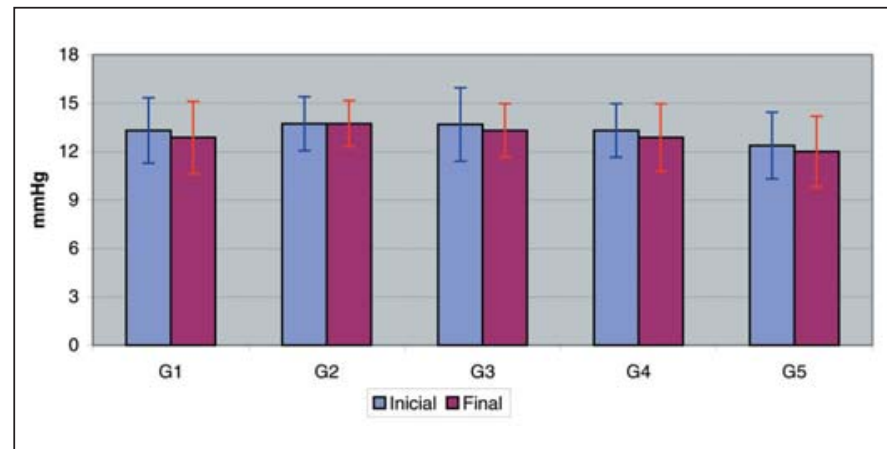

Gráfico 3 - Valores das médias da Po $(\mathrm{mmHg})$ iniciais e finais nos cinco grupos do teste $\mathrm{T} 3$ e respectivos intervalos de um desvio padrão

(T1), exercício (T2) e exercício sob leitura (T3) não houve diferença estatisticamente significante entre os grupos: G1, G2, G3, G4 e G5.

A avaliação do comportamento da Po em indivíduos com hipertensão arterial sistêmica, diabetes mellitus e indivíduos normais mostra os respectivos resultados: hipertensos sem retinopatia (média $\mathrm{Po}=16,10 \mathrm{mmHg}$ ), e hipertensos com retinopatia (média $\mathrm{Po}=16,33 \mathrm{mmHg}$ ) e diabéticos e hipertensos com retinopatia (média $\mathrm{Po}=16,95 \mathrm{mmHg}$ ), concluindo-se que o valor médio da Po é progressivamente mais elevado conforme o tempo de evolução da doença hipertensiva e quando associada à diabetes mellitus ${ }^{(9)}$.

Neste estudo ao ser comparado portadores de glaucoma ou hipertensão ocular com indivíduos normais observou-se o mesmo comportamento da Po em ambos os grupos. Provavelmente não houve alteração da Po inicial e final pelo fato de estarem usando um colírio antiglaucomatoso, portanto estavam na condição de "normais". É possível que o comportamento da Po fosse diferenciado, caso tais pacientes portadores de glaucoma ou hipertensão ocular, não fizessem uso de colírio antiglaucomatoso.

O presente estudo observou grupos sem e com hipertensão arterial sistêmica usuários de análogos de prostaglandinas, prostamidas, ou beta-bloqueadores em ambientes de leitura e exercício. Os resultados demonstraram que não houve diferença significante entre os tipos de testes de leitura (T1), exercício (T2) e exercício sob leitura (T3) nos diferentes grupos, não sendo a leitura e o exercício físico fatores que interferiram negativamente no tratamento de pacientes com glaucoma primário de ângulo aberto ou hipertensos oculares com e sem hipertensão arterial sistêmica.

Exercícios utilizando treinamento de força em homens e mulheres com Po normal fisicamente ativos, apresentaram uma redução moderada da Po, sendo recomendada cautela na generalização dos resultados com relação à recomendação de exercícios de força para pacientes com Po elevada. Indivíduos com glaucoma deverão realizar exercícios de intensidades moderadas $^{(8)}$. Na presente pesquisa, não houve diferença significante ao realizar os testes estabelecidos. Talvez se os exercícios fossem mais intensos, o resultado poderia ser diferente, ou seja, encontrar diminuição significante entre a Po inicial e final. Em contrapartida a leitura e o nível de concentração seriam prejudicados em função do nível de esforço requerido.

O protocolo utilizado possibilitou que todos os participantes realizassem a leitura durante o exercício de forma tranqüila, o que um protocolo com um percentual mais elevado para o exercício poderia comprometer a qualidade de concentração e disposição para o teste.

Os resultados do presente estudo mostram que as drogas que estavam sendo usadas para controlar a PA não influenciaram nos valores da Po ao realizar exercícios.

Há ainda um desconhecimento sobre os mecanismos e definição mais precisa sobre a ação do glaucoma primário de ângulo aberto, sendo uma neuropatia com variações com relação a nível da Po. A associação do glaucoma com outras doenças requer uma atenção particular principalmente quando associada a hipertensão arterial sistêmica. A hipertensão necessita ser controlada, no entanto a redução abrupta pode gerar um impacto destruindo o nervo óptico ${ }^{(14)}$. Na presente pesquisa não foi objetivo estudar o efeito hipotensor das drogas utilizadas para tratar a hipertensão arterial e o impacto destas sobre o nervo óptico. No entanto, seria interessante verificar se a prática de exercícios diários poderia afetar, a longo prazo, o nervo óptico em pacientes portadores de glau- 
coma primário de ângulo aberto e hipertensão arterial controlada através de medicamentos.

Um estudo avaliando a Po e leitura, exercício e exercício sob leitura utilizando um protocolo de exercícios moderados com homens entre 18 e 35 anos fisicamente ativos e saudáveis e sem comprometimento ocular, avaliando a Po antes e após cada teste, demonstrou uma redução estatisticamente não significante da $\mathrm{Po}^{(15)}$.

Não foram encontrados na literatura pesquisada, estudos referentes à verificação de Po de pacientes com glaucoma primário de ângulo aberto ou hipertensão ocular em ambientes de exercício sob leitura.

Os resultados desta pesquisa demonstraram que usuários de análogos de prostaglandinas, prostamidas ou beta-bloqueadores com e sem hipertensão arterial sistêmica responderam da mesma maneira aos testes de leitura, exercício e exercício sob leitura, não sendo a diferença entre os grupos estatisticamente significativa. Não foi encontrado trabalhos na literatura pesquisada para comparação desses achados.

A partir dos resultados do presente estudo, oftalmologistas poderão responder aos pacientes portadores de glaucoma ou hipertensão ocular sobre a prática de leitura e exercício individualmente ou concomitantemente. Os resultados demonstraram que estes, poderão ler e fazer exercícios caso a Po esteja controlada, assim como a PA nos casos de hipertensão arterial sistêmica. Com exceção do grupo G2 (hipertensos sistêmicos utilizando beta-bloqueador) para o teste específico de exercício sob leitura no qual a Po inicial foi igual a final, houve uma redução da Po final em todos os grupos nos três testes.

\section{CONCLUSÃO}

Sendo o glaucoma uma doença crônica, durante seu tratamento é necessário um acompanhamento e pesquisas que tornem este quadro mais esclarecedor. Segundo os resultados do presente estudo, ler e fazer exercícios físicos em bicicleta ergométrica, não são fatores de agravos à Po de indivíduos com glaucoma primário de ângulo aberto ou hipertensos oculares com e sem hipertensão arterial sistêmica. Sugerem-se novos estudos que observem o comportamento da Po de portadores de glaucoma primário de ângulo aberto praticando leitura enquanto realizam exercícios físicos.

\section{ABSTRACT}

Purpose: To check intraocular pressure (IOP) in individuals using prostaglandin, prostamide or beta-blocker analogues, who sustain either primary open angle glaucoma or ocular hypertension after reading, exercise or exercise combined with reading. Methods: 40 individuals (79 eyes), subdivided in to five groups: G1 (with arterial hypertension and either glaucoma or ocular hypertension, all users of prostaglandin or pros- tamide analogues); G2 (with arterial hypertension and either glaucoma or ocular hypertension, all users of beta-blockers); G3 (not sustaining arterial hypertension but suffering from either glaucoma or ocular hypertension, all users of prostaglandin or prostamide analogues); G4 (not sustaining arterial hypertension but suffering from either glaucoma or ocular hypertension, all users of beta-blockers) and G5 (not sustaining arterial hypertension and also not suffering from either glaucoma or ocular hypertension) had their intraocular pressure checked before and after undergoing reading, exercise, and exercise combined with reading. Each type of test was conducted in a different day, always in the afternoon. Results: No significant statistical difference has been noticed between the initial and final intraocular pressure mean in the different groups, when reading, performing exercises or exercises combined with reading. Conclusion: To read and to work out - either separately or jointly - does not pose an aggravating factor to the intraocular pressure of patients with primary open glaucoma or ocular hypertension, using prostaglandin, prostamide or beta-blocker analogues.

Keywords: Glaucoma, open angle; Intraocular pressure/drug therapy; Reading; Exercise; Ocular hypertension; Prostaglandins/therapeutic use; Tonometry, ocular

\section{REFERÊNCIAS}

1. Blake J, Horgan T, Carroll P, Stokes J, Fitzpatrick P. Effect of accommodation of the lens on ocular pressure. Ir J Med Sci. 1995 Oct-Dec;164(4): 269-70.

2. Cassidy L, Delaney Y, Fitzpatrick P, Blake J. Effect of accommodation on intraocular pressure in glaucomatous eyes. Ir J Med Sci. 1998;167(1):17-8.

3. Shapiro A, Wolf E, Ferber I, Merin S. The effect of physical activity on the intraocular pressure of glaucomatous patients. Eur J Appl Physiol Occup Physiol. 1983;52(1):136-8.

4. Qureshi IA. Effects of mild, moderate and severe exercise on intraocular pressure of sedentary subjects. Ann Hum Biol. 1995;22(6):545-53.

5. Kiuchi Y, Mishima HK, Hotehama Y, Furumoto A, Hirota A, Onari K. Exercise intensity determines the magnitude of IOP decrease after running. Jpn J Ophthalmol. 1994;38(2):191-5.

6. Raposo-Filho A. Variações da pressão intra-ocular em indivíduos submetidos ao teste cicloergométrico [tese]. São Paulo: Escola Paulista de Medicina; 1992.

7. Chromiak JA, Abadie BR, Braswell RA, Koh YS, Chilek DR. Resistance training exercises acutely reduce intraocular pressure in physically active men and women. J Strength Cond Res. 2003;17(4):715-20.

8. Sakata K, Maia M, Matsumoto L, Oyamaguchi EK, Carvalho ACA, Knoblauch N, Oliveira Filho AG. Estudo do comportamento da pressão intra-ocular em pacientes diabéticos, hipertensos e normais (projeto Glaucoma). Arq Bras Oftalmol. 2000;63(3):219-22.

9. Hodapp E, Parrigh II RK, Anderson DR. Clinical decisions in glaucoma. St. Louis: C. V. Mosby; 1993.

10. Barros TL de. O Programa das 10 Semanas. Uma proposta para trocar gordura por músculos e saúde. São Paulo: Manole; 2002. 41p.

11. Myers KJ. The effect of aerobic exercise on intraocular pressure. Invest Ophthalmol. 1974;13(1):74-6.

12. Shapiro A, Shoenfeld Y, Shapiro Y. The effect of standardized submaximal work load on intraocular pressure. Br J Ophtalmol. 1978;62(10):679-81.

13. Leighton DA. Effect of walking on the ocular tension in open-angle glaucoma. Br J Ophthalmol. 1972;56(2):126-30.

14. Lecture D. Glaucoma: facts and facies. R Coll Ophtalmol. 1996;10:295-301.

15. Medina AMC, Bezerra AJC. Verificação da pressão intra-ocular em indivíduos submetidos a exercício cicloergométrico sob leitura. Rev Bras Ciên Mov. 2001; 9(4):33-9. 